\title{
Framework for selecting Assistive Technology User-Participation Methods.
}

\author{
E.A. Draffan, Abi James, Mike Wald and Amal Idris
}

Corresponding author: EA Draffan ead@ecs.soton.ac.uk

Submitted: 27 January 2016,

Accepted: $17^{\text {th }}$ March 2016

Journal of Assistive Technologies 10(2):92-101 · June 2016

DOI: 10.1108/JAT-01-2016-0007

\section{Authors Draft}

\section{Abstract}

\section{Purpose}

A number of participatory research methodologies can be used to assist with developing assistive technologies. These methods vary in the amount that users lead and contribute to the work.

Selecting the correct method can be important to ensure the overall success of the project and the engagement of users. This paper explores factors that can impact on the degree of user participation.

\section{Design/methodology/approach}

The paper considers whether criteria, that might influence assistive technology selection made during an assessment of need, review or purchasing process, could also be used to clarify the appropriate strategies for user involvement when developing assistive technologies. It outlines how this approach has been applied to two research and development projects which aimed to improve assistive technology provision within niche markets.

\section{Findings}

The paper demonstrates that it is possible to apply a decision making process to selecting the best participatory research method, based on factors affecting assistive technology need. it reports on the outcomes of the user participation in the two research and development projects and discusses how this design approach has been applied to a third project.

\section{Originality/value}

By examining a possible framework for identifying appropriate user participation approaches, this paper will aid those designing research and development assistive technology projects, whilst encouraging user participation within similar projects.

\section{Keywords:}

Participatory design, assistive technology, participation, research methodology, disability, user involvement

\section{Article Classification}

Research Paper 


\title{
Framework for selecting Assistive Technology User-Participation Methods.
}

\author{
E.A. Draffan, Abi James, Mike Wald and Amal Idris
}

Corresponding author: EA Draffan ead@ecs.soton.ac.uk

Submitted: 27 January 2016,

Accepted: $17^{\text {th }}$ March 2016

Journal of Assistive Technologies 10(2):92-101 · June 2016

DOI: 10.1108/JAT-01-2016-0007

\section{Authors Draft}

\section{Introduction}

The design of Assistive Technology (AT) tools, whether related to content that will be used in other programs or actual applications, has been shown in the past to benefit from user involvement where there is an understanding regarding the type of participatory methodology used in the process (Newell \& Gregor, 2000). Inclusive or user-centred design based on human computer interactions, as originally described by Norman \& Draper (1986), has been introduced to the assistive technology world by Newell and Gregor (2000) as 'user sensitive inclusive design'. This collaborative approach is one with which the authors are familiar, having researched participatory methodologies with users of assistive technology for two previous research projects (Seale et al, 2008; Cudd et al, 2011). However, the AT projects discussed in this paper were very different in nature, requiring those involved to engage with "the relationship between the functionality of users and the environment in which they may operate" as suggested by Newell (1995) in very particular ways. One project had a specific cultural element and the other a specialised subject element, although both involved disabled users working with speech and language therapists or teachers and academics mainly in educational settings.

Project 1 involved the development of a set of culturally sensitive pictograms for an online Arabic symbol dictionary. Each pictogram or symbol was designed to be used alongside other alternative and augmentative communication (AAC) symbol sets for those who have communication and literacy difficulties in the Gulf region. Project 2 focussed on a mathematical notation reading program for use by those who may have Dyscalculia or print disabilities that impacted on the ability to read equations. Both projects needed to offer workable and sustainable technology solutions and involve potential users as part of the design process.

One benefit of involving potential users is that as participants they may take ownership of the research (Cornwell \& Jewkes 1995:1667) to encourage improved outcomes and facilitate increased uptake of the AT content and tools being developed. However, given the specialist nature of the applications, it was recognised that there might be a limited pool of collaborators. The participatory framework used in the previous projects (based on work by Radermacher, 2006), did not provide any AT centred criteria to help with the choice of an appropriate userparticipation methodology. Therefore, the teams working jointly on both projects considered whether criteria, that might influence AT selection made during an assessment of need, review or purchasing process, could also be used to clarify the appropriate strategies for user involvement when developing AT. These criteria encompass rather more than the functional and environmental issues suggested by Newell (1995) and some may be recognized as part of a 'Matching Person and Technology' process (Scherer, 1998). The combined criteria were adapted 
to influence the type of participatory approach that was to be taken for each project and eventually to act as a guide for a third project. Project 3 was an online application to support potential users of Project 1 and was developed by a new AT developer, also requiring a user participation design approach.

\section{Brief description of the Projects}

\section{Project 1}

Speech and language therapists and specialist teachers in Qatar have often had to use AAC symbols designed in the United States of America or United Kingdom with westernised imagery and Arabic translated labels. Although the development of bespoke symbols for individual AAC users and for those using symbols to support literacy skills will always remain important, there was a general feeling that a set of freely available symbols that covered major topics in a manner that was localized to fit with the culture, home, educational and work settings as well as Arabic linguistic needs would be welcomed. Whilst the project team in the University of Southampton had some knowledge around the use of symbols for communication purposes, there was an enormous gap in their knowledge about how professionals working in Qatar coped with their particular user requirements and how they would be able to participate in the process of designing the symbols.

\section{Project 2}

Project 2, known as the STEMReader project, involved the design and development of an application that could read aloud English mathematical notation embedded within documents and electronic materials. Once again the University project team had experts in the field of accessibility, mathematics and Computer Sciences, but the tool involved a new concept for supporting users with print impairments to access maths equations with voice output and graphical representations. In order for the new technology to be accepted, it was vital that users were able to identify the perceived benefits of the tool and found it easy to use (Venkatesh \& Davis, 2000). User-participation was therefore critical to inform the design and application of the tool and the same could be said to be true for the symbols and their presentation in Project 1.

Would the constraints of developing content for use in other AT tools require different participatory methodologies when compared to developing a software application? Would it be possible to rank the constraints to support the degree of user participation? Newell and Gregor (2000) highlight the tensions that can exist when involving disabled users and experts in research that involves the development of assistive technologies. Not only are there ethical considerations but also the very special considerations of a few users that may impact on others when considering requirements. On the one hand they talk about 'test pilots' and on the other hand 'user panels', 'case studies' and 'individual users' who work with them to evaluate the outcomes of the designed artefacts. Project 1 and 2 required the use of technology and contained content that needed to be viewed, understood and then evaluated against a set of criteria, but there the similarities ended. Each project involved the need for pilot studies, beta testing and user group discussions but they had very different cultural settings and language issues. The users and those supporting them also required different skills and abilities. One project was about content acceptance for communication, the other about the use of an application to generate content in 
an accessible manner. There needed to be a clearer understanding of the constraints that would impact on the amount of possible user participation and the methodology employed to achieve successful outcomes.

\section{Methodology for selecting user participation strategy}

The research teams undertook a two phase process prior to making decisions as to which participatory methodology would best suit their projects. The initial phase was to go through a series of assistive technology criteria to ensure all aspects had been taken into account when working with users and experts in the field. The second phase involved reviewing the various participatory methods categorised by Radermacher (2006) before making a final decision as to which would be the best match for the project requirements.

\section{Checking Assistive Technology Criteria prior to choosing a Participatory Methodology}

As has been mentioned Scherer (1998), discusses user characteristics, functionality, environment and many more criteria when thinking about AT choices. In order to consider the participatory approach that needed to be taken for the two projects, the researchers incorporated some of Scherer's criteria into a model that they called STREET as seen in Figure 1. STREET represents the personal Strengths that a user may have to undertake the Tasks proposed; the Resources required plus the Expertise they bring with them; the Environment in which they may be working and finally the Tools that may be needed. The criteria are made up of several components which are not mutually exclusive. Typically, when selecting suitable AT for a user, the practitioner considers all the potential variables that may be influenced by the user's Strengths, Tasks, Resources, Expertise and Environment in order to arrive at a suitable collection of Tools. To isolate those variables that could constrain user participation in Project 1 and 2, the authors reversed this process as the 'Tools' were fixed by the intended outcome of the project, namely a tool using symbols to aid communication in the case of Project 1 and a mathematical notation reader for Project 2.

\begin{tabular}{|c|c|c|c|c|c|}
\hline Strengths: & Tasks: & Resources: & Expertise: & Environment: & Tools: \\
\hline $\begin{array}{l}\text { Visual, } \\
\text { Auditory } \\
\text { Kinaesthetic } \\
\text { Dexterity \& } \\
\text { Mobility } \\
\text { Cognition and } \\
\text { processing } \\
\text { Receptive } \\
\text { /Expressive } \\
\text { Language } \\
\text { Health }\end{array}$ & $\begin{array}{l}\text { Reading \& } \\
\text { understanding } \\
\text { information } \\
\text { Writing } \\
\text { Organisation \& } \\
\text { Planning } \\
\text { Communication } \\
\text { Memory \& Recall } \\
\text { Time, Money \& } \\
\text { Numeracy } \\
\text { Daily Living }\end{array}$ & $\begin{array}{l}\text { Financial } \\
\text { Procurement } \\
\text { Timeframe } \\
\text { Training } \\
\text { Peer support } \\
\text { Support } \\
\text { Professionals } \\
\text { Technical } \\
\text { support }\end{array}$ & $\begin{array}{l}\text { Prior knowledge \& } \\
\text { experience } \\
\text { Metacognition } \\
\text { Personal } \\
\text { Preferences/ } \\
\text { Strategies } \\
\text { Technology skills } \\
\text { Perceived benefit/ } \\
\text { Confidence }\end{array}$ & $\begin{array}{l}\text { Workplace/Study } \\
\text { / Living } \\
\text { environment } \\
\text { System/Operating } \\
\text { Environment } \\
\text { Accessibility/ } \\
\text { Constraints } \\
\text { Compatibility } \\
\text { Security \& IT } \\
\text { Policies }\end{array}$ & $\begin{array}{l}\text { Communication } \\
\text { Text to speech / } \\
\text { eReading } \\
\text { Speech recognition } \\
\text { Word processing \& } \\
\text { proofing tools } \\
\text { Recording/Capturing } \\
\text { Graphical mapping/ } \\
\text { Planning } \\
\text { Reminders } \\
\text { Calculators \& Maths } \\
\text { Alarms \& environmental } \\
\text { controls }\end{array}$ \\
\hline
\end{tabular}

Figure 1. STREET Strengths, Tasks, Resources, Expertise, Environment, Tools 
In the case of Project 1 the experts needed to be able to share their knowledge about the vocabularies used and their views about symbol choices, (for instance comparing a woman dressed in Arabic clothing with a woman in westernised dress) and AAC users needed to have the ability to indicate their symbol preferences. All users, family, carers and experts needed to be able to see the symbols and to indicate choices in order to record a decision in a similar fashion to using an AAC device or communication board. The task was one of symbol based communication in a supported sense and although it was proposed that symbol labels would be available in both Arabic and English, reading was not a requirement. In terms of resources, the project was to take place over 3 years with 30 months of symbol development and was intended to produce a set of resources with a Creative Commons (CC-BY-4.0) licence, so that the participants could share the project outputs and develop them further in the future. There were financial constraints related to travel and support for participants with face to face meetings and voting sessions for symbol acceptance. However, minimal technical support and training were required. The team needed to be aware of the environment in which the symbols would be used as well as any issues around IT support, security, compatibility and accessibility constraints as the symbols were to be provided for download as well as to be used for printed materials. This also meant that the online tools that would be used for storing information (symbols with their metadata) and text to speech in Arabic and English had to be carefully chosen in advance of any development with features that would fit the participants' requirements.

Project 2 was based on an earlier proof-of-concept project and was awarded funding through a competitive process to further develop the STEMReader tool involving users and stakeholders. The funding bodies for the project wished to ensure user participation was included within its development but also decided upon a number of design criteria which would affect the level of the user participation. One constraint was that STEMReader had to run on a Windows operating system and needed to be tested in post-16 education institutions (from vocational courses to degree levels) as well as the workplace. The project was to run for 18 months and, as development would be happening in parallel to testing, it was anticipated that technical resources from the project team would be required to assist with testing while the tool was still in development. Reviewing the criteria required for Project 2, it was clear that the strengths included auditory and kinaesthetic abilities, but not necessarily visual, as the application would be designed to be used by those with visual impairments. The task was one of reading and assimilating information and this could occur within a teacher-led session as part of a learning activity or user-led when trying to read and assimilate information from books, documents and internet-based resources. Some experience in reading mathematical notation, such as fractions was considered essential.

Previous studies have demonstrated that first impressions often influence feedback from users and, in particular, users with little experience of speech feedback are often preoccupied with the clarity and intelligibility of computer generated speech (McCarthy et al, 2013). While it would have been advantageous for users to be confident with the use of text to speech output, Project 2 intended to design a novel approach to using this type of AT in maths and only a few users would have been familiar with these approaches. The authors were particularly aware that prior experience of text to speech could significantly impact users' participation and perception during any evaluation process and it was important to capture and analyse feedback from users where AT skill levels and prior experiences differed.

So it can be seen from the various criteria that a number of constraints have appeared. In Project 1 it was mainly about the strengths and /or experience of the participant, linked to their ability to make choices about symbols and available resources such as time and funding in order to attend 
sessions to choose symbols for the Arabic Symbol Dictionary. In Project 2 decisions about the operating system and presentation mode of the application had to be made prior to any participation taking place and the range of environments and levels of expertise would constrain the degree of user participation as well as the numbers able to beta test or take part in trials.

In order to evaluate the constraints on user-participation identified through the STREET analysis, each one was ranked on a scale of 1-5 (Figure 2). This clearly showed that there were less constraints on user participation within Project 1 than Project 2. The ranking was based on the number of components within the STREET criteria that had to be built into the interactions with participants.

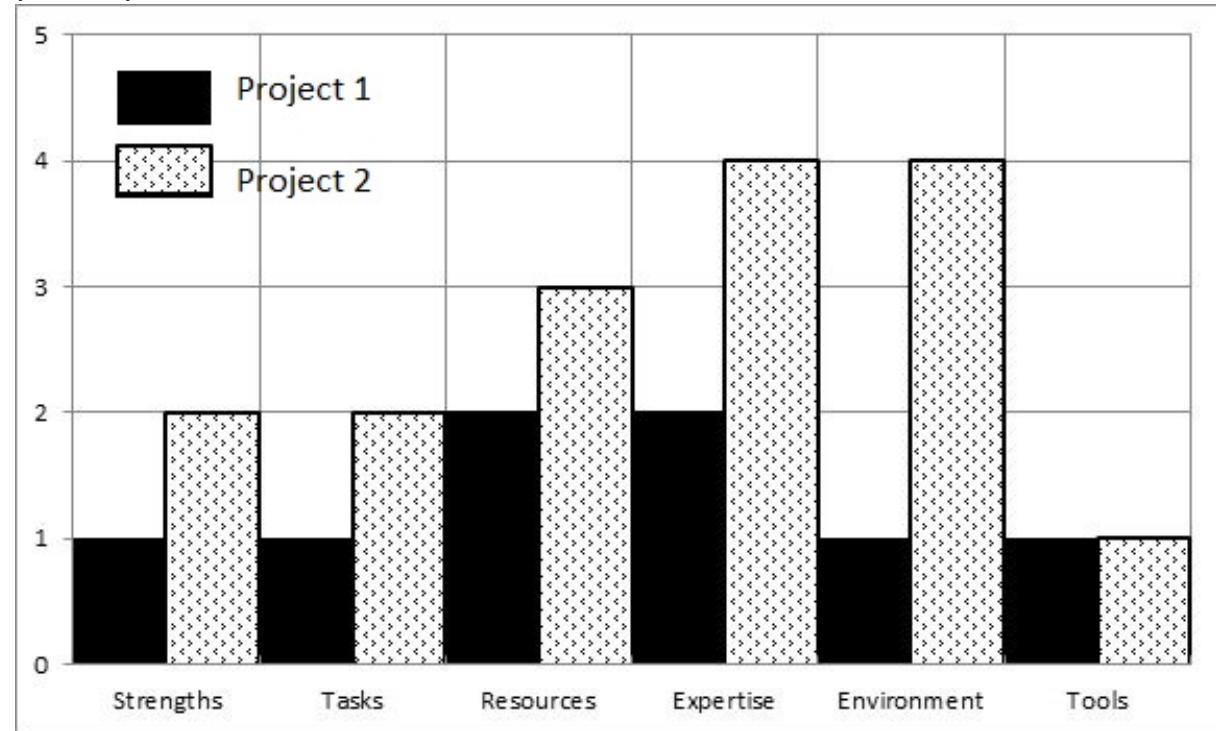

Figure 2. Constraints ranked on a scale of 1-5. In Project 1, resources and expertise ranked 2. In Project 2, resources ranked 3 ; expertise and environment ranked 4

\section{Participatory methodology choices}

Ward \& Trigler 2001 (Pg 58) stated that, in order to maximise user-participation, there needed to be clear aims and objectives as to how this could be achieved and how the roles of the participants and experts could be seen in terms of their involvement. Radermacher (2006) identified from Fajerman and Treseder, (2000), that there were six degrees of user participation ranging from no involvement down to total involvement:

1. Non-involvement: the project is designed and run by the researcher.

2. Consulted and informed: researcher consults users. Opinions are taken seriously but do not alter the project process.

3. Assigned but informed: participants volunteer and are involved with the project. Opinions inform an iterative design process.

4. Researcher-initiated, shared decisions with participants: participants volunteer and are involved with the project design from the initial stages.

5. Participant-initiated, shared decisions with researcher: participants initiate and design the project, with the assistance of a researcher.

6. Participant-initiated and directed: participants initiate, design and direct the project.

In the case of both Project 1 and 2 engaging with participants was not just about encouraging ownership and uptake of the AT research outcomes, but also to fill the knowledge gaps between the designer/researchers' expertise and those of the anticipated users. The wider the gap, the more important it was to have a high degree of user-participation. In Project 1 , it had been 
identified that, while the research team and the participants were knowledgeable about the technology and its application in their individual environments; understanding and communicating cultural, social and linguistic differences was key to the project's success. In Project 2, the team were keen for users to inform the product design and concept throughout the project but both developers and participants had knowledge about the cultural, social and linguistic environments.

It was felt that neither project could entirely be controlled by the participants nor could there be total 'Non-involvement' of users. However, in keeping with Walmsley's (2004) suggestion when working with learning disabled users "in contrast to emancipatory research, in participatory research, non-disabled people have an enduring role." So each project considered the 'assigned but informed' approach, where the researcher may have already defined the research project but the participants are fully aware of the involvement required and their opinions are recognised as being important for a successful outcome and the 'consulted and informed approach' where the participants' views are taken into account but there is less 'hands on' user involvement. The final option could be 'researcher-initiated' rather than 'participant-initiated' with all the decisions being shared with participants. In this case the participants would not only be involved at the outset with planning and decision making but they would also influence aspects of the running of the project to ensure successful outcomes and uptake of the end product where appropriate. This left 3 possible approaches for the user participation for the two projects, each combining researcher and user input.

- Consulted and informed

- Assigned but informed

- Researcher-initiated, shared decisions with participants

\section{Applying STREET analysis to user-participatory methods}

A pragmatic approach was needed in order to make the right decisions for each project to overcome "conflicting pressures on researchers ..." (Chappell, 2000). The STREET analysis highlighted the fact that some decisions would need to be made prior to inviting potential participants to join the research due to resource related constraints, such as financial, technical and time. Project 1 had the potential to fail to meet the expectations of potential users if the gap in local knowledge by one side of the team was not bridged, but it had time and a minimal requirement for participants to have high levels of technological or specialist expertise with these constraints only ranking 2 out of 5 in the analysis. However, by adopting a 'researcher-initiated, shared decisions with participants' approach, choices about task design, the symbol acceptance and the way technology could be used to support interactions with end users and other stakeholders could become an iterative process (Nielsen, 1993) with each re-evaluation improving outputs.

Project 2 not only had to overcome complex accessibility and technology issues, but also had additional resource constraints (ranking 3 ) with a short technology development timeline. It needed to engage participants in a number of environments with a range of expertise (both ranking 4). These constraints would limit engagement with decision making so the 'researcherinitiated, shared decisions with participants' approach appeared to be impractical. The project considered the option of 'consulted and informed' versus 'assigned but informed'. Whilst the former approach may have been a more efficient use of resources and could have involved more users, it provided no means of assuring voluntary participation throughout the project, nor did it allow for informed participatory feedback. The 'assigned but informed' approach enabled the 
research team to engage with a range of users adapting the intended application to fit their requirements based on feedback received during each testing phase.

\section{Results}

Project 1 engaged participants in discussions about the design of the symbol acceptance systems and presentation models. Initial trials showed that both users and experts required a very simplistic flash card type system to make choices with an easy 5-point Likert scale for the experts and an even easier 3-point scale for AAC users (Draffan et al, 2015). Both options allowed for additional comments and could be used online and on paper. When discussing the idea of compulsory open rather than closed questions all participants showed a preference for optional comments and check boxes linked to statements due to user strengths, time constraints and language issues.

Participants engaged in four voting sessions during the first year of the project, reviewing 60-65 symbols each time. Initial sessions had 63 participants providing 2341 votes for 65 symbols and those that were found to be unsuitable (with a score of less than 3.5) were re-designed based on comments received. These symbols were then re-evaluated in later voting sessions to ensure overall acceptance. Participants went on to state which symbols should be prioritised with requests for religious imagery, local dress and settings as well as offering advice about the dictionary website design and delivery methods for supporting resources. As the project progressed more requests came from other stakeholders, such as companies who wished to use the symbols on their devices and needed to download them in specific formats. There were also requests to include the symbols in a local Qatari publication demonstrating that user participation can lead to a wider stakeholder buy-in to a project.

Project 2 used the 'assigned but informed' approach for user participation. The project linked phases of user participation to its development plan. Each phase of user testing focussed on specific aspects of the application. Participants for each phase were assigned based on their strengths and the environmental factors impacting their use of technology. User participation was undertaken through the use of semi-structured interviews to introduce users to the software and feedback was captured through open-ended questions. Users were then observed using the application and making use of 'think-aloud' procedures (McDonald et. al, 2012). In addition, users were encouraged to complete a post-testing survey to reflect on their experiences through a combination of open-ended question and 5-point Likert scales.

The first phase of testing for Project 2 focussed on feedback from students at university who were experienced in the use of AT which provided input into developing a new interface. The second phase of testing allowed the project to gain feedback on the interface changes and specific reading tasks identified as needing further participatory input during the first phase of testing. In the final phase participants with a wide range of skill levels took part in testing and were given the opportunity to try out new methods of interacting with the tool from other applications, based on previous feedback. Each of the stages involved between 10 and 20 volunteers, from at least 2 organisations, with diverse experiences and needs. This approach provided the opportunity to trial experimental and prototype user interface features. It also encouraged engagement within the stakeholder community in over 30 organisations. 
As a result of the previous two projects, the authors felt confident in applying the STREET criteria to inform the degree of user participation possible in a third project working with a new AT developer. The developer only had six months to design and deliver an online application for symbol adaptations, but was keen to involve a System Usability Scale (Brooke, 1996). The criteria indicated that Project 3 had to take place with limited time for training participants, minimal technical support and a very short development phase with more hours spent on project reporting rather than testing. The application would be used in the workplace, clinics and schools, had to be fully accessible and compatible with a range of browsers. The participants needed to be able to manipulate symbols using technology and have some knowledge of basic image adaptation tools. The participants also had to be experts already providing symbols for AAC users with a "full understanding of the process..." (Radermacher, 2006). STREET ranking levels reached at least 3 in all the criteria signifying considerable limitations on the amount of user participation and a reduced chance of altering the overall project process. However, the criteria highlighted that a 'consulted and informed' participatory methodology was possible and would still allow for some user involvement where opinions were taken seriously despite the small scale of the project.

\section{Discussion}

Making decisions about the degree of AT user participation required when developing AT software necessitates careful planning. It cannot be 'ad hoc' in its approach as issues may be overlooked that may impact on outcomes. These may be very different from other types of user participation requiring extended time allowances for user interactions with additional support along with a recognition of particular user strengths and expertise in terms of access to technology.

A systematic approach that allows the researcher to check a series of criteria such as those offered by STREET may encourage the developer to look more carefully at individual and group needs as well as providing a better insight into potential overheads. However, as has been illustrated, if choices are made with full awareness of the number of constraints involved even limited user participation can be achieved within a development cycle.

On reflection it can be seen that Project 1 provided the researchers with a much greater insight into user requirements for every aspect of the development but this took time. Had the result been to use an 'assigned but informed' approach, it might have made the decision making processes speedier and it may have been possible to create more symbols. But a reduced degree of participation could have led to the development of redundant symbols or ones that would have been inappropriate. Supporting resources may also have been unsuitable with decreased stakeholder uptake.

Project 2 had initially hoped to gather feedback through continuous engagement with a specific user group using a 'researcher-initiated, shared decisions with participants' approach. However, the STREET analysis identified that the 'assigned but informed' approach was more appropriate. allowing for iterative testing within a shorter timeframe and with less resources. The approach was also a better match for the agile development methodology used in the project. By assigning users to testing phases, the project was able to ensure that feedback was received at an appropriate stage of the development of the application, as well as enabling the testing of new features as they were developed. Volunteers became 'knowledgeable participants' and supported usability, compatibility, accessibility and functionality testing. The users contributed to focus group meetings and design discussions around the various elements of the application. The participatory method chosen also allowed users with differing levels of expertise to take part. In 
addition, whilst the number of participants was low in comparison to Project 1, direct user engagement enabled the team to capture a large amount of information on how users were able to interact with the application.

Finally, Project 3 demonstrated that the framework could be applied to other types of AT technology projects even if they were small scale. Constraints could be highlighted and made obvious to the developer, so that hopes to have a user led project could be channelled into a successful methodology with the resources available.

\section{Conclusion}

As a framework to support the use of a particular participatory methodology, suitable for AT developments, the STREET analysis with the ranking of constraints linked to Radermacher's (2006) degrees of participant involvement, was intended to be a useful decision making exercise. It allowed the authors to apply a systematic process to planning user participation across three different projects. This increased the feeling of confidence about their choice of participatory methodology in relation to the potential user's strengths, tasks, resources, expertise, environment and tools needed. The process appears to have also resulted in positive interactions between participants and researchers that enabled the work on the projects to better take into account user requirements.

Just as the choices made when matching AT to user needs have to be bespoke for successful outcomes and user uptake, so to do the choices made regarding the degree of participant involvement with AT research and development. The higher the number of components that are ranked as constraints, the less likely it appears that research methodologies involving participants can be initiated by or driven by them. While this may be mainly due to those components such as time and finance that can be judged as resource constraints at the start of a research and development project, the STREET analysis has shown the importance of participants' expertise and the environment in which they are situated; whether this is related to physical settings or specialist, cultural, social and linguistic environments. It should also be noted that even with the third project where there were a considerable number of constraints it was still possible to develop a user-participation strategy. Therefore, it is felt that this approach has highlighted the fact that careful analysis of all the components involved in the suggested framework can lead to better AT participatory design and research methodologies with potential users informing best practice.

\section{References}

Brooke, J. (1996), "SUS-A quick and dirty usability scale", Usability evaluation in industry, Vol. 189 No. 194, pp. 4-7.

Chappell, A (2000), "Emergence of participatory methodology in learning difficulty research: understanding the context" British Journal of Learning Disabilities, Vol. 28, pp. 38-43

Cornwall, A. \& Jewkes, R. (1995), "What is Participatory Research?", Social Science and Medicine, vol. 41 , no. 12 , pp. 1667-1676. 
Cudd, P., Draffan, E. A., Wald, M., \& Lee, S. (2011), "Designing the marriage of Open Innovation and User Participation", Assistive Technology Research Series, Vol. 29, pp. 354-361.

Draffan, E., Wald, M., Halabi, N., Kadous, A., Idris, A., Zeinoun, N., Banes, D. \& Lawand, D. (2015), "A Voting System for AAC Symbol Acceptance", Proceedings of the 17th International ACM SIGACCESS Conference on Computers \& Accessibility (ASSETS '15). ACM, New York, NY, USA, pp. 371-372. DOI=http://dx.doi.org/10.1145/2700648.2811374

Fajerman, L.,\& Treseder, P. (2000), “Children are service users too: a guide to consulting children and young people", Save The Children, London.

McCarthy, T., Pal, J., \& Cutrell, E. (2013), "The "voice" has it: screen reader adoption and switching behavior among vision impaired persons in India", Assistive Technology, Vol. 25 No. 4, pp. 222-229. doi:10.1080/10400435.2013.768719

McDonald, S., Edwards, H. M., \& Zhao, T. (2012), "Exploring think-alouds in usability testing: An international survey", Professional Communication, IEEE Transactions on, Vol. 55 No. 1, pp. 2-19.

Newell A.F. (1995). "Extra-ordinary human-computer interaction", in Edwards A. (Ed.), Extraordinary human-computer interaction, Cambridge Series On Human-Computer Interaction, Vol. 7. Cambridge University Press, New York, NY, USA pp. 3-18

Newell, A. F., \& Gregor, P. (2000), "User sensitive inclusive design - in search of a new paradigm", Proceedings on the 2000 conference on Universal Usability, ACM, pp. 39-44.

Nielsen, J. (1993). "Iterative User Interface Design". IEEE Computer vol.26 no.11 pp 32-41.

Norman, D. A. \& Draper, S. W. (Editors) (1986), User-Centered System Design: New Perspectives on Human-Computer Interaction. Lawrence Earlbaum Associates, Hillsdale, NJ.

Radermacher, H. (2006), "Participatory Action Research With People With Disabilities: Exploring Experiences Of Participation”, Doctoral Thesis. Victoria University, Melbourne, Australia.

Scherer, M.J. (1998), The Matching Person \& Technology (MPT) Model Manual, 3rd ed., The Institute for Matching Person \& Technology, Inc, Webster, NY

Seale, J., Wald, M., \& Draffan, E. (2008), “Exploring the technology experiences of disabled learners in higher education: challenges for the use and development of participatory research methods", Journal of Assistive Technologies, Vol. 2 No. 3, pp. 4-15.

Venkatesh, V., \& Davis, F. D. (2000), "A theoretical extension of the technology acceptance model: Four longitudinal field studies", Management science, Vol. 46 No.2, pp. 186-204.

Walmsley, J. (2004), "Inclusive learning disability research: the (nondisabled) Researcher's role", British Journal of Learning Disabilities, Vol. 32 No. 2, pp. 65-71.

Ward, K., \& Trigler, J. S. (2001), "Reflections on participatory action research with people who have developmental disabilities", Mental Retardation, Vol. 39, N. 1 pp. 57-59. 
